\title{
A New Class of Meromorphic Functions Involving the Polylogarithm Function
}

\author{
Khadeejah Rasheed Alhindi and Maslina Darus \\ School of Mathematical Sciences, Faculty of Science and Technology, Universiti Kebangsaan Malaysia (UKM), \\ 43600 Bangi, Selangor Darul Ehsan, Malaysia
}

Correspondence should be addressed to Maslina Darus; maslina@ukm.edu.my

Received 10 May 2014; Revised 25 July 2014; Accepted 26 July 2014; Published 11 August 2014

Academic Editor: Jacek Dziok

Copyright $(9) 2014$ K. R. Alhindi and M. Darus. This is an open access article distributed under the Creative Commons Attribution License, which permits unrestricted use, distribution, and reproduction in any medium, provided the original work is properly cited.

We introduce a new operator $D_{c} f(z)$ associated with polylogarithm function. By making use of the new operator, we define a certain new class of meromorphic functions and discussed some important properties of it.

\section{Introduction}

Historically, the classical polylogarithm function was invented in 1696, by Leibniz and Bernoulli, as mentioned in [1]. For $|z|<1$ and $c$ a natural number with $c \geq 2$, the polylogarithm function (which is also known as Jonquiere's function) is defined by the absolutely convergent series:

$$
\operatorname{Li}_{c}(z)=\sum_{k=1}^{\infty} \frac{z^{k}}{k^{c}} .
$$

Later on, many mathematicians studied the polylogarithm function such as Euler, Spence, Abel, Lobachevsky, Rogers, Ramanujan, and many others [2], where they discovered many functional identities by using polylogarithm function. However, the work employing polylogarithm has been stopped many decades later. During the past four decades, the work using polylogarithm has again been intensified vividly due to its importance in many fields of mathematics, such as complex analysis, algebra, geometry, topology, and mathematical physics (quantum field theory) [3-5]. In 1996, Ponnusamy and Sabapathy discussed the geometric mapping properties of the generalized polylogarithm [6]. Recently, $\mathrm{Al}$-Shaqsi and Darus generalized Ruscheweyh and Salagean operators, using polylogarithm functions on class $\mathscr{A}$ of analytic functions in the open unit disk $\mathbb{U}=\{z:|z|<1\}$. By making use of the generalized operator they introduced certain new subclasses of $\mathscr{A}$ and investigated many related properties [7]. A year later, same authors again employed the $n$th order polylogarithm function to define a multiplier transformation on the class $\mathscr{A}$ in $\mathbb{U}[8]$.

To the best of our knowledge, no research work has discussed the polylogarithm function in conjunction with meromorphic functions. Thus, in this present paper, we redefine the polylogarithm function to be on meromorphic type.

Let $\Sigma$ denote the class of functions of the form

$$
f(z)=\frac{1}{z}+\sum_{k=0}^{\infty} a_{k} z^{k},
$$

which are analytic in the punctured open unit disk

$$
\mathbb{U}^{*}:=\{z: z \in \mathbb{C}, 0<|z|<1\}=: \mathbb{U} \backslash\{0\} .
$$

A function $f(z)$ in $\Sigma$ is said to be meromorphically starlike of $\operatorname{order} \delta$ if and only if

$$
\Re\left\{-\frac{z f^{\prime}(z)}{f(z)}\right\}>\delta ; \quad\left(z \in \mathbb{U}^{*}\right),
$$

for some $\delta(0 \leq \delta<1)$. We denote by $\Sigma^{*}(\delta)$ the class of all meromorphically starlike order $\delta$. Furthermore, a function $f(z)$ in $\Sigma$ is said to be meromorphically convex of order $\delta$ if and only if

$$
\mathfrak{R}\left\{-1-\frac{z f^{\prime \prime}(z)}{f^{\prime}(z)}\right\}>\delta ; \quad\left(z \in \mathbb{U}^{*}\right),
$$


for some $\delta(0 \leq \delta<1)$. We denote by $\Sigma_{K}(\delta)$ the class of all meromorphically convex order $\delta$. For functions $f \in \Sigma$ given by (2) and $g \in \sum$ given by

$$
g(z)=\frac{1}{z}+\sum_{k=0}^{\infty} b_{k} z^{k}
$$

we define the Hadamard product (or convolution) of $f$ and $g$ by

$$
(f * g)(z)=\frac{1}{z}+\sum_{k=0}^{\infty} a_{k} b_{k} z^{k}
$$

Let $\Sigma_{p}$ be the class of functions of the form

$$
f(z)=\frac{1}{z}+\sum_{k=0}^{\infty} a_{k} z^{k} ; \quad a_{k} \geq 0,
$$

which are analytic and univalent in $\mathbb{U}^{*}$.

Liu and Srivastava [9] defined a function $h_{p}\left(\alpha_{1}, \ldots, \alpha_{q}\right.$; $\left.\beta_{1}, \ldots, \beta_{s} ; z\right)$ by multiplying the well-known generalized hypergeometric function ${ }_{q} F_{s}$ with $z^{-p}$ as follows:

$$
\begin{aligned}
h_{p} & \left(\alpha_{1}, \ldots, \alpha_{q} ; \beta_{1}, \ldots, \beta_{s} ; z\right) \\
& =z^{-p}{ }_{q} F_{s}\left(\alpha_{1}, \ldots, \alpha_{q} ; \beta_{1}, \ldots, \beta_{s} ; z\right),
\end{aligned}
$$

where $\alpha_{1}, \ldots, \alpha_{q} ; \beta_{1}, \ldots, \beta_{s}$ are complex parameters and $q \leq$ $s+1, p \in \mathbb{N}$.

Analogous to Liu and Srivastava work [9] and corresponding to a function $\Phi_{c}(z)$ given by

$$
\Phi_{c}(z)=z^{-2} \operatorname{Li}_{c}(z)=\frac{1}{z}+\sum_{k=0}^{\infty} \frac{1}{(k+2)^{c}} z^{k},
$$

we consider a linear operator $\Omega_{c} f(z): \Sigma \rightarrow \Sigma$ which is defined by the following Hadamard product (or convolution):

$$
\begin{aligned}
\Omega_{c} f(z) & =\Phi_{c}(z) * f(z) \\
& =\frac{1}{z}+\sum_{k=0}^{\infty} \frac{1}{(k+2)^{c}} a_{k} z^{k} .
\end{aligned}
$$

Next, we define the linear operator $D_{c} f(z): \Sigma \rightarrow \Sigma$ as follows:

$$
\begin{aligned}
D_{c} f(z) & =\left\{\Omega_{c} f(z)-\frac{1}{2^{c}} a_{0}\right\} \\
& =\frac{1}{z}+\sum_{k=1}^{\infty} \frac{1}{(k+2)^{c}} a_{k} z^{k} .
\end{aligned}
$$

Now, by making use of the operator $D_{c} f(z)$, we define a new subclass of functions in $\Sigma_{p}$ as follows.

Definition 1. For $\alpha>1$ and $0<\beta \leq 1$, let $N_{c}(\alpha, \beta)$ denote a subclass of $\Sigma$ consisting functions of form (2) satisfying the condition that

$$
\mathfrak{R}\left\{z D_{c} f(z)-\alpha z^{2}\left(D_{c} f(z)\right)^{\prime}\right\}>\beta ; \quad\left(z \in \mathbb{U}^{*}\right),
$$

where $D_{c} f(z)$ is given by (12). Furthermore, we say that a function $f \in N_{c, p}(\alpha, \beta)$, whenever $f(z)$ is of form (8).
In the following sections, we investigate coefficient inequalities, extreme points, radii of starlikeness and convexity of order $\delta$, and integral means inequalities for the new class $N_{c, p}(\alpha, \beta)$.

\section{Coefficient Inequalities}

The following theorem gives a necessary and sufficient condition for a function $f$ to be in the class $N_{c, p}(\alpha, \beta)$.

Theorem 2. Let $f \in \Sigma_{p}$ given by (8). Then $f \in N_{c, p}(\alpha, \beta)$ if and only if

$$
\sum_{k=1}^{\infty} \frac{(\alpha k-1)}{(k+2)^{c}} a_{k} \leq 1+\alpha-\beta .
$$

Proof. Suppose that $f \in N_{c, p}(\alpha, \beta)$. Then

$$
\begin{aligned}
\mathfrak{R}\left\{z\left(\frac{1}{z}+\sum_{k=1}^{\infty} \frac{1}{(k+2)^{c}} a_{k} z^{k}\right)\right. \\
\left.-\alpha z^{2}\left(\frac{-1}{z^{2}}+\sum_{k=1}^{\infty} \frac{k}{(k+2)^{c}} a_{k} z^{k-1}\right)\right\} \\
=\mathfrak{R}\left\{1+\alpha-\sum_{k=1}^{\infty} \frac{\alpha k-1}{(k+2)^{c}} a_{k} z^{k+1}\right\}>\beta .
\end{aligned}
$$

If we choose $z$ to be real and letting $z \rightarrow 1$, we get

$$
1+\alpha-\sum_{k=1}^{\infty} \frac{(\alpha k-1)}{(k+2)^{c}} a_{k}>\beta,
$$

which is equivalent to (14). Conversely, let us suppose that assertion (14) holds true.

Then we can write

$$
\begin{aligned}
& \left|z D_{c} f(z)-\alpha z^{2}\left(D_{c} f(z)\right)^{\prime}\right| \\
& =\left|1+\alpha-\sum_{k=1}^{\infty} \frac{\alpha k-1}{(k+2)^{c}} a_{k} z^{k+1}\right| \\
& \quad \geq 1+\alpha-\left|\sum_{k=1}^{\infty} \frac{\alpha k-1}{(k+2)^{c}} a_{k} z^{k+1}\right| \\
& >\beta .
\end{aligned}
$$

Hence, $f \in N_{c, p}(\alpha, \beta)$. Finally, we note that inequality (14) is sharp; the extremal function is

$$
f(z)=\frac{1}{z}+\frac{(1+\alpha-\beta)(3)^{c}}{(\alpha-1)} z ; \quad \alpha>1 .
$$

\section{Extreme Points}

In this section, we determine the extreme points for functions in the class $N_{c, p}(\alpha, \beta)$. 
Theorem 3. Let $f_{0}(z)=1 / z$ and

$$
f_{k}(z)=\frac{1}{z}+\frac{(1+\alpha-\beta)(k+2)^{c}}{(\alpha k-1)} z^{k} ; \quad(k=1,2, \ldots) .
$$

Then, $f \in N_{c, p}(\alpha, \beta)$ if and only if it can be represented in the form

$$
f(z)=\sum_{k=0}^{\infty} \lambda_{k} f_{k}(z) ; \quad\left(\lambda_{k} \geq 0, \sum_{k=0}^{\infty} \lambda_{k}=1\right)
$$

Proof. Let $f(z)=\sum_{k=0}^{\infty} \lambda_{k} f_{k}(z), \lambda_{k} \geq 0, k=0,1,2, \ldots$, $\sum_{k=0}^{\infty} \lambda_{k}=1$. Then, we have

$$
\begin{aligned}
f(z) & =\sum_{k=0}^{\infty} \lambda_{k} f_{k}(z) \\
& =\lambda_{0} f_{0}(z)+\sum_{k=1}^{\infty} \lambda_{k} f_{k}(z) \\
& =\frac{1}{z}+\sum_{k=1}^{\infty}\left\{\lambda_{k} \frac{(1+\alpha-\beta)(k+2)^{c}}{(\alpha k-1)}\right\} z^{k} .
\end{aligned}
$$

Therefore,

$$
\begin{gathered}
\sum_{k=1}^{\infty} \frac{(\alpha k-1)}{(k+2)^{c}} \frac{(1+\alpha-\beta)(k+2)^{c}}{(\alpha k-1)} \lambda_{k} \\
\quad=(1+\alpha-\beta) \sum_{k=1}^{\infty} \lambda_{k} \\
=(1+\alpha-\beta)\left(1-\lambda_{0}\right) \\
\leq(1+\alpha-\beta) .
\end{gathered}
$$

Hence, by Theorem 2, $f \in N_{c, p}(\alpha, \beta)$.

Conversely, we suppose that $f \in N_{c, p}(\alpha, \beta)$, since

$$
a_{k} \leq \frac{(1+\alpha-\beta)(k+2)^{c}}{(\alpha k-1)} ; \quad k \geq 1
$$

We set

$$
\lambda_{k}=\frac{(\alpha k-1)}{(1+\alpha-\beta)(k+2)^{c}} a_{k} ; \quad k \geq 1,
$$

and $\lambda_{0}=1-\sum_{k=1}^{\infty} \lambda_{k}$. Then we have

$$
\begin{aligned}
f(z) & =\sum_{k=0}^{\infty} \lambda_{k} f_{k}(z) \\
& =\lambda_{0} f_{0}(z)+\sum_{k=1}^{\infty} \lambda_{k} f_{k}(z) .
\end{aligned}
$$

The results follow.

\section{Radii of Meromorphic Starlikeness and Meromorphic Convexity}

Theorem 4. Let $f \in N_{c, p}(\alpha, \beta)$. Then $f$ is meromorphically starlike of order $\delta(0 \leq \delta<1)$ in the disk $|z|<r_{1}$, where

$$
r_{1}=\inf _{k}\left[\left(\frac{1-\delta}{k+2-\delta}\right) \frac{(\alpha k-1)}{(1+\alpha-\beta)(k+2)^{c}}\right] ; \quad(k \geq 1) \text {. }
$$

The result is sharp for the extremal function $f(z)$ given by (19).

Proof. It is sufficient to show that

$$
\left|\frac{z f^{\prime}(z)}{f(z)}+1\right|<1-\delta
$$

which easily follows from (4), since

$$
\begin{aligned}
\left|\frac{z f^{\prime}(z)}{f(z)}+1\right| & =\left|\frac{\sum_{k=1}^{\infty}(k+1) a_{k} z^{k+1}}{1+\sum_{k=1}^{\infty} a_{k} z^{k+1}}\right| \\
& \leq \frac{\sum_{k=1}^{\infty}(k+1)\left|a_{k}\right||z|^{k+1}}{1-\sum_{k=1}^{\infty}\left|a_{k}\right||z|^{k+1}} .
\end{aligned}
$$

Considering that $\sum_{k=1}^{\infty}\left|a_{k}\right|<1$, the above expression is less than $1-\delta$ if and only if

$$
\begin{aligned}
\sum_{k=1}^{\infty}(k & +1)\left|a_{k}\right||z|^{k+1} \\
& \leq(1-\delta)\left(1-\sum_{k=1}^{\infty}\left|a_{k}\right||z|^{k+1}\right),
\end{aligned}
$$

or

$$
\sum_{k=1}^{\infty} \frac{k+2-\delta}{1-\delta}\left|a_{k}\right||z|^{k+1} \leq 1
$$

By Theorem 2, we have

$$
\sum_{k=1}^{\infty} \frac{(\alpha k-1)}{(k+2)^{c}(1+\alpha-\beta)} a_{k} \leq 1
$$

then, (27) holds true if

$$
\frac{k+2-\delta}{1-\delta}|z|^{k+1}<\frac{(\alpha k-1)}{(k+2)^{c}(1+\alpha-\beta)} ; \quad k \geq 1
$$

which is equivalent to

$$
|z|^{k+1}<\frac{1-\delta}{k+2-\delta} \frac{(\alpha k-1)}{(1+\alpha-\beta)(k+2)^{c}},
$$

which yields the starlikeness of the family and completes the proof. 
Theorem 5. Let $f \in N_{c, p}(\alpha, \beta)$. Then $f$ is meromorphically convex of order $\delta(0 \leq \delta<1)$ in the disk $|z|<r_{2}$, where

$$
r_{2}=\inf _{k}\left[\left(\frac{1-\delta}{k+2-\delta}\right) \frac{(\alpha k-1)}{k(1+\alpha-\beta)(k+2)^{c}}\right] ; \quad(k \geq 1) .
$$

The result is sharp for the extremal function $f(z)$ given by

$$
f_{k}(z)=\frac{1}{z}+\frac{k(1+\alpha-\beta)(k+2)^{c}}{(\alpha k-1)} z^{k} ; \quad(k=1,2, \ldots) .
$$

Proof. By using the technique employed in the proof of Theorem 4, we can show that

$$
\left|\frac{z f^{\prime \prime}(z)}{f^{\prime}(z)}+2\right|<1-\delta
$$

for $|z|<r_{2}$, and prove that the assertion of the theorem is true.

\section{Integral Means Inequalities}

Let $f(z)$ and $g(z)$ be analytic in $\mathbb{U}^{*}$. Then the function $f(z)$ is said to be subordinate to $g(z)$ in $\mathbb{U}^{*}$, written by

$$
f(z) \prec g(z) ; \quad\left(z \in \mathbb{U}^{*}\right),
$$

if there exists a function $w(z)$ which is analytic in $\mathbb{U}^{*}$ with $w(0)=0$ and $|w(z)|<1$ with $z \in \mathbb{U}^{*}$ and such that $f(z)=g(w(z))$ for $z \in \mathbb{U}^{*}$. From the definition of the subordinations, it is easy to show that subordination (37) implies that

$$
f(0)=g(0), \quad f\left(\mathbb{U}^{*}\right) \subset g\left(\mathbb{U}^{*}\right) .
$$

Theorem 6 (see [10]). If $f$ and $g$ are any two functions, analytic in $\mathbb{U}$, with $f \prec g$, then, for $\eta>0$ and $z=r e^{i \theta} ;(0<r<1)$,

$$
\int_{0}^{2 \pi}|f(z)|^{\eta} d \theta \leq \int_{0}^{2 \pi}|g(z)|^{\eta} d \theta
$$

Theorem 7. Suppose $f \in N_{c, p}(\alpha, \beta)$ and $f_{k}$ is defined by

$$
f_{k}(z)=\frac{1}{z}+\frac{(1+\alpha-\beta)(k+2)^{c}}{(\alpha k-1)} z^{k} ; \quad(k=1,2, \ldots) .
$$

If there exists an analytic function $w(z)$ such that

$$
[w(z)]^{k+1}=\frac{(\alpha k-1)}{(1+\alpha-\beta)(k+2)^{c}} \sum_{k=1}^{\infty} a_{k} z^{k+1}
$$

then, for $z=r e^{i \theta}$ and $(0<r<1)$,

$$
\int_{0}^{2 \pi}\left|f\left(r e^{i \theta}\right)\right|^{\eta} d \theta \leq \int_{0}^{2 \pi}\left|f_{k}\left(r e^{i \theta}\right)\right|^{\eta} d \theta ; \quad(\eta>0) .
$$

Proof. We need to show that

$$
\begin{aligned}
& \int_{0}^{2 \pi}\left|1+\sum_{k=1}^{\infty} a_{k} z^{k+1}\right|^{\eta} d \theta \\
& \quad \leq \int_{0}^{2 \pi}\left|1+\frac{(1+\alpha-\beta)(k+2)^{c}}{(\alpha k-1)} z^{k+1}\right|^{\eta} d \theta .
\end{aligned}
$$

From Theorem 6, it suffices to prove that

$$
1+\sum_{k=1}^{\infty} a_{k} z^{k+1} \prec 1+\frac{(1+\alpha-\beta)(k+2)^{c}}{(\alpha k-1)} z^{k+1} .
$$

If we set $w$ such that

$$
[w(z)]^{k+1}=\frac{(\alpha k-1)}{(1+\alpha-\beta)(k+2)^{c}} \sum_{k=1}^{\infty} a_{k} z^{k+1},
$$

we get

$$
1+\sum_{k=1}^{\infty} a_{k} z^{k+1}=1+\frac{(1+\alpha-\beta)(k+2)^{c}}{(\alpha k-1)}[w(z)]^{k+1} .
$$

Clearly, $w(0)=0$; then from Theorem 2 we can write

$$
\begin{aligned}
& |w(z)|^{k+1} \\
& \quad=\left|\frac{(\alpha k-1)}{(1+\alpha-\beta)(k+2)^{c}} \sum_{k=1}^{\infty} a_{k} z^{k+1}\right| \\
& \quad \leq \frac{(\alpha k-1)}{(1+\alpha-\beta)(k+2)^{c}} \sum_{k=1}^{\infty}\left|a_{k}\right||z|^{k+1} \\
& \quad<|z|<1 .
\end{aligned}
$$

That completes the proof.

\section{Conflict of Interests}

The authors declare that they have no conflict of interests.

\section{Authors' Contribution}

Both authors read and approved the final paper.

\section{Acknowledgment}

The authors would like to thank the center of research and instrumentation (CRIM), National University of Malaysia (UKM), for sponsoring this work under Grant code GUP2013-004.

\section{References}

[1] C. I. Gerhardt and G. W. Leibniz, Mathematische Schriften III/1, Georg Olms, New York, NY, USA, 1971.

[2] L. Lewin, Polylogarithms and Associated Functions, NorthHolland, Oxford, UK, 1981. 
[3] A. B. Goncharov, "Polylogarithms in arithmetic and geometry," in Proceedings of the International Congress of Mathematicians, pp. 374-387, Zürich, Switzerland, August 1994.

[4] L. Lewin, "Structural properties of polylogarithms, math, surveys and monographs," American Mathematical Society, vol. 73, 1991.

[5] S. Oi, "Gauss hypergeometric functions, multiple polylogarithms, and multiple zeta values," Publications of the Research Institute for Mathematical Sciences, vol. 45, no. 4, pp. 981-1009, 2009.

[6] S. Ponnusamy and S. Sabapathy, "Polylogarithms in the theory of univalent functions," Results in Mathematics, vol. 30, no. 1-2, pp. 136-150, 1996.

[7] K. Al Shaqsi and M. Darus, "An operator defined by convolution involving the polylogarithms functions," Journal of Mathematics and Statistics, vol. 4, no. 1, pp. 46-50, 2008.

[8] K. Al-Shaqsi and M. Darus, "A multiplier transformation defined by convolution involving nth order polylogarithm functions," International Mathematical Forum, vol. 4, no. 37-40, pp. 1823-1837, 2009.

[9] J. Liu and H. M. Srivastava, "Classes of meromorphically multivalent functions associated with the generalized hypergeometric function," Mathematical and Computer Modelling, vol. 39, no. 1, pp. 21-34, 2004.

[10] J. E. Littlewood, "On inequalities in the theory of functions," Proceedings of the London Mathematical Society, vol. 23, no. 1, pp. 481-519, 1925. 


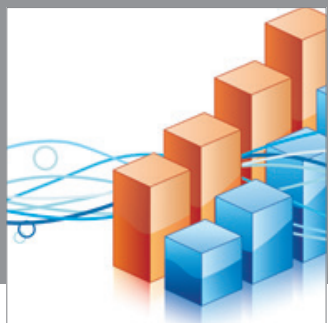

Advances in

Operations Research

mansans

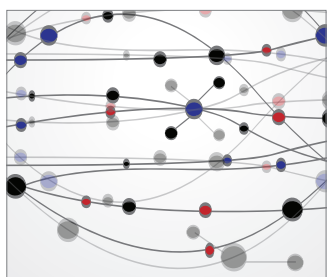

The Scientific World Journal
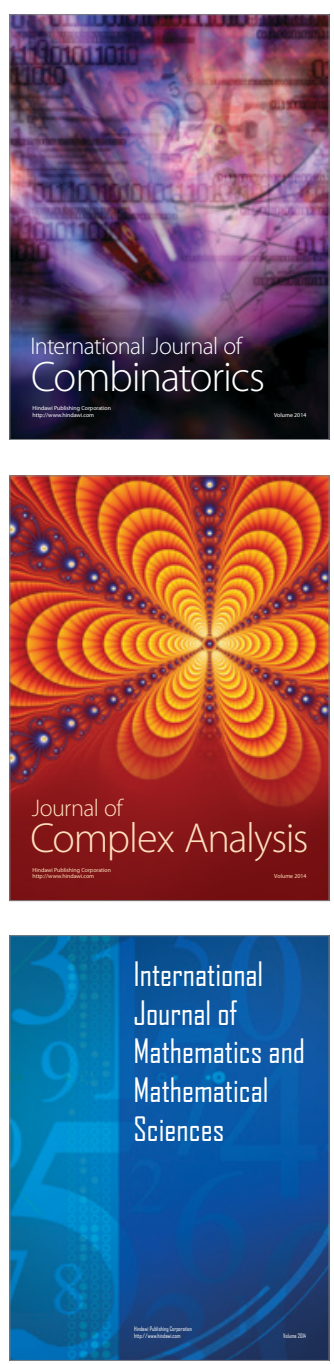
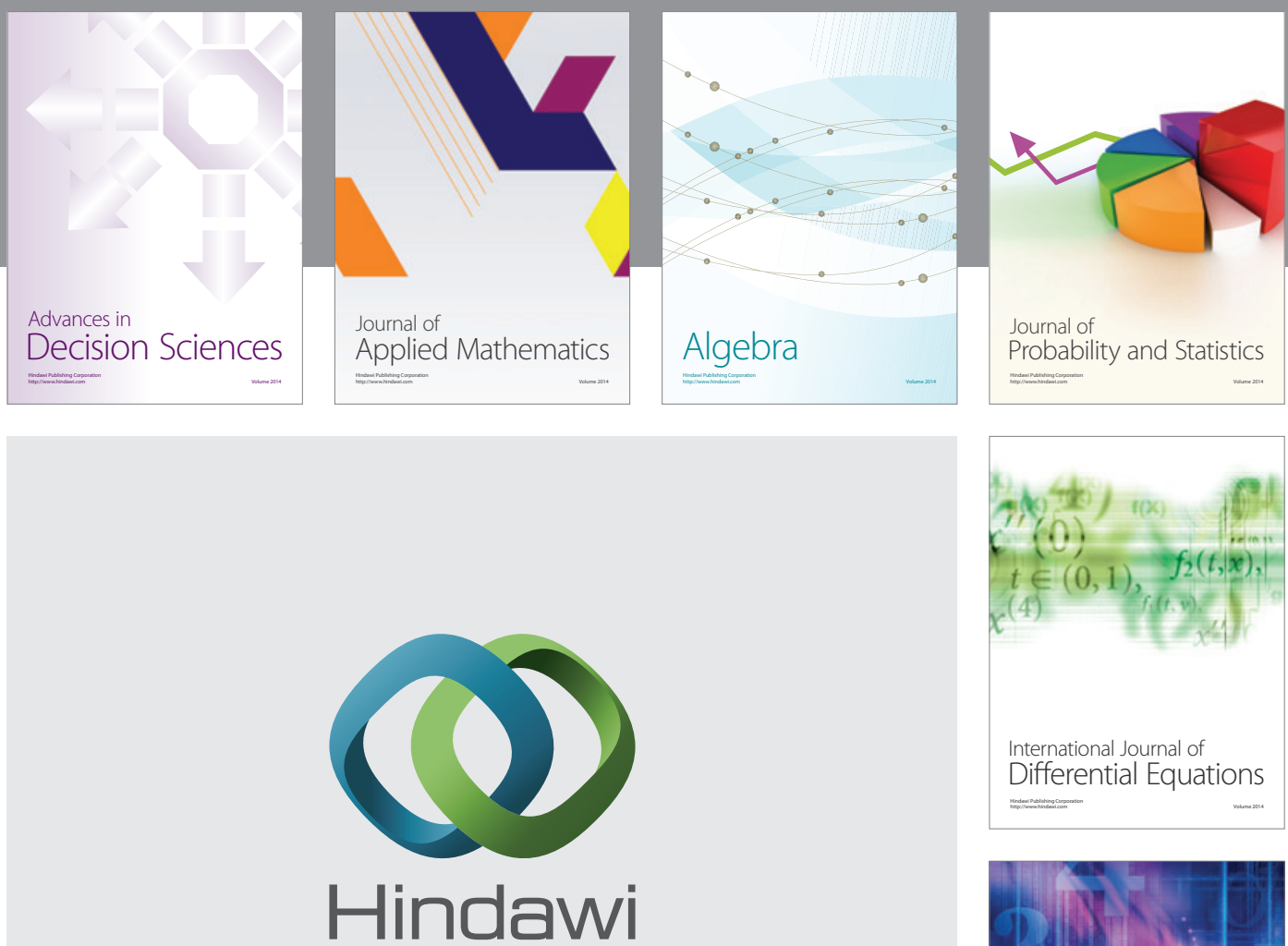

Submit your manuscripts at http://www.hindawi.com
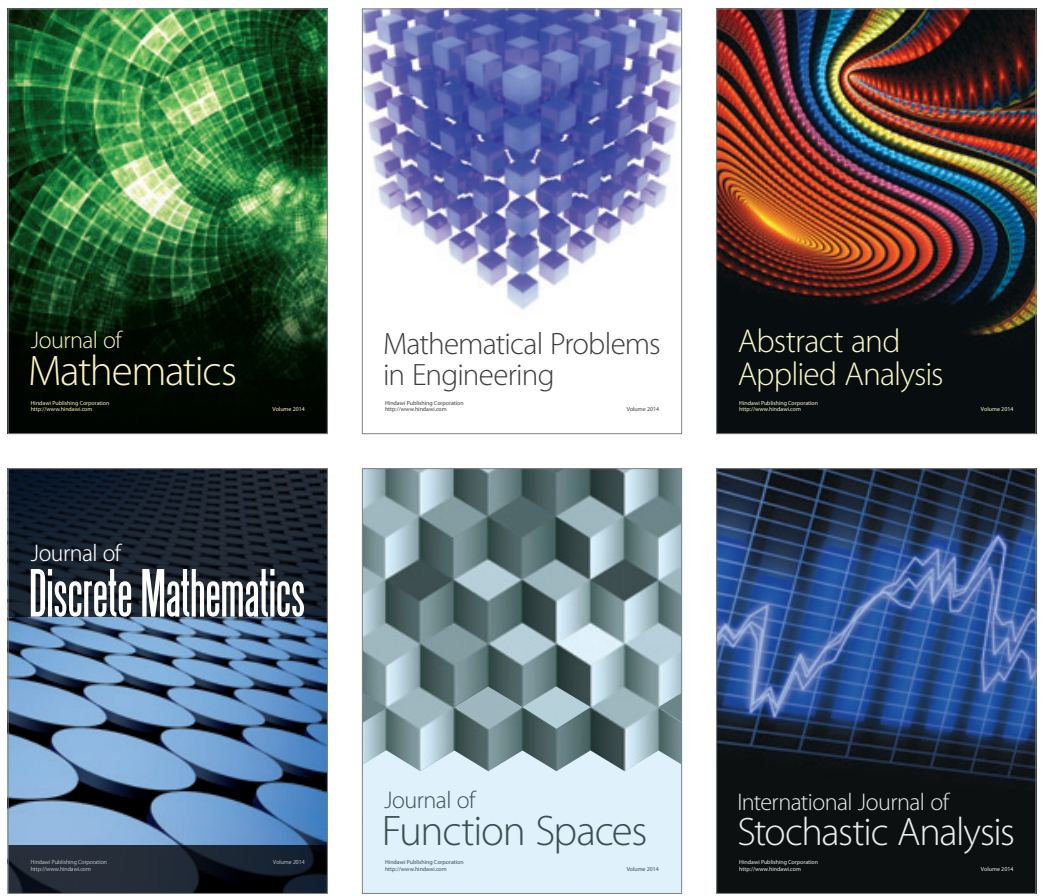

Journal of

Function Spaces

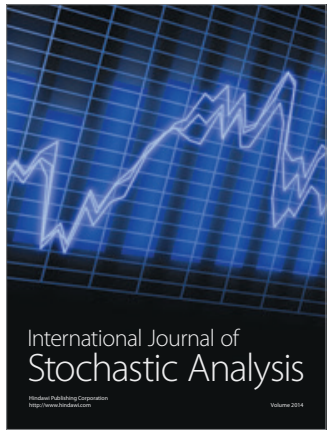

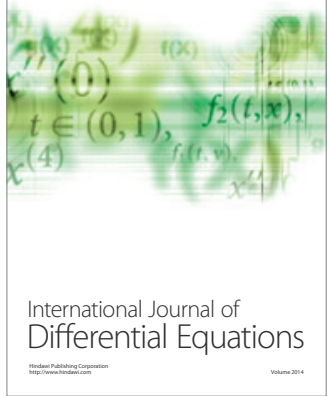
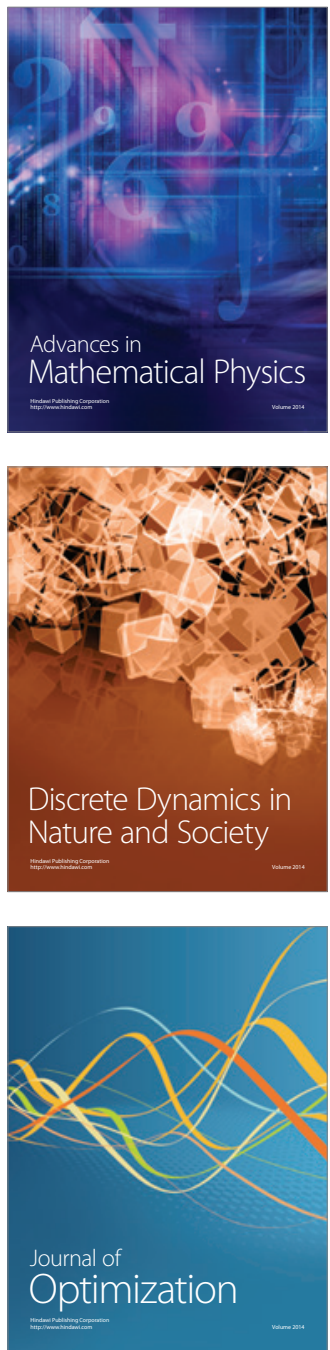